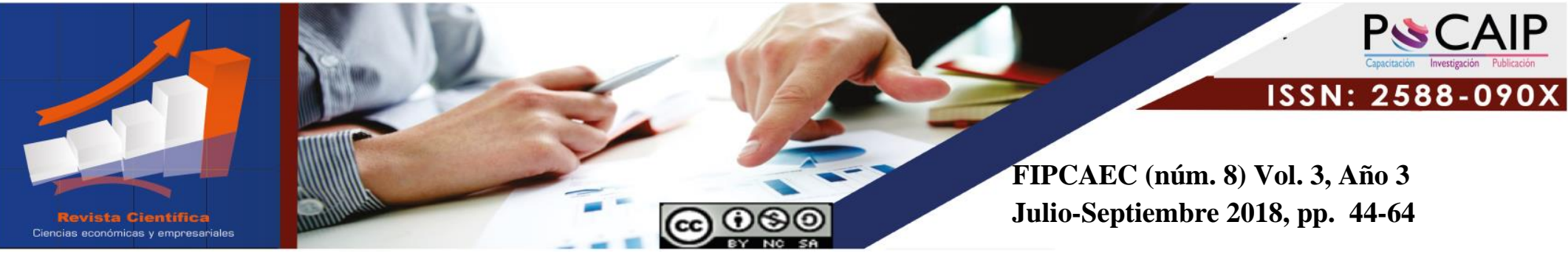

DOI: DOI 10.23857/fipcaec.v3i8.57

\title{
Gestión Empresarial: una paradigma del siglo XXI
}

\section{Business Management: a paradigm of the 21st century}

\section{Gestão Empresarial: um paradigma do século XXI}

\author{
Michael Josué Suárez-Espinar ${ }^{1}$ \\ josue201114v@gmail.com \\ https://orcid.org/0000-0003-1423-5081
}

Correspondencia: josue201114v@gmail.com

* Recepción: 23/ 04/ 2018 * Aceptación: 26/06/ 2018 *Publicación: 15 /07/ 2018

$1 \quad$ Investigador Independiente, Manta, Ecuador. 


\title{
Resumen
}

La gestión empresarial es un rasgo principal que debe tener todo hombre de negocios. La misma dentro del sistema empresarial es un instrumento fundamental para su progreso económico, ya que representa la dinámica de una economía de mercado, que es la que garantiza que la oferta cubra a la demanda en distintas actividades productivas, creando conjuntamente procedimientos que hacen que los costos bajen continuamente y beneficien al consumidor. El capital se ubica siempre a producir aquello que la sociedad demanda para la satisfacción de determinadas necesidades, circunstancia que da cuenta de la eficiencia del mercado en lo que concierne a la retribución de recursos. Las funciones principales que la integran son: planificación, organización, dirección, conducción liderazgo y control. Esta se orienta y se mide a través de herramientas e indicadores de gran valor para su progreso. Con una adecuada gestión la empresa tendrá excelentes resultados y nuevas metas.

Palabras clave: Gestión empresarial; importancia; funciones; herramientas.

\begin{abstract}
Business management is a main feature that every businessman must have. The same within the business system is a fundamental instrument for its economic progress, since it represents the dynamics of a market economy, which is what guarantees that supply meets demand in different productive activities, jointly creating procedures that make costs go down continuously and benefit the consumer. Capital is always located to produce what society demands for the satisfaction of certain needs, a circumstance that accounts for the efficiency of the market with regard to the retribution of resources. The main functions that integrate it are: planning, organization, direction, leadership leadership and control. This is oriented and measured through tools and indicators of great value for your progress. With proper management the company will have excellent results and new goals.
\end{abstract}

Keywords: Business management; importance; functions; tools. 


\section{Resumo}

Gerenciamento de negócios é uma característica principal que todo empresário deve ter. $\mathrm{O}$ mesmo no sistema de negócios é um instrumento fundamental para o seu progresso econômico, pois representa a dinâmica de uma economia de mercado, que garante que a oferta atenda à demanda em diferentes atividades produtivas, criando conjuntamente procedimentos que os custos diminuem continuamente e beneficiam o consumidor. O capital está sempre localizado para produzir o que a sociedade exige para a satisfação de certas necessidades, circunstância que explica a eficiência do mercado no que diz respeito à retribuição de recursos. As principais funções que o integram são: planejamento, organização, direção, liderança, liderança e controle. Isso é orientado e medido através de ferramentas e indicadores de grande valor para o seu progresso. Com gestão adequada, a empresa terá excelentes resultados e novas metas.

Palavra-chave: Gestão de negócios; importância; funções; ferramentas.

\section{Introducción}

En el diccionario la palabra gestión proviene del Latín gestīo. Hace referencia a la administración de recursos, tanto en una institución estatal o privada, para lograr los objetivos propuestos por la misma. (Mora, Duran, \& Zambrano, 2016).

Se conceptualiza en las literaturas estudiadas, que gestión es la acción y el efecto de gestionar y administrar. Se puede decir que, una gestión es una diligencia, entendida como un trámite necesario para conseguir algo o resolver un asunto, habitualmente de carácter administrativo o que conlleva documentación, otra referencia; es también un conjunto de acciones u operaciones relacionadas con la administración y dirección de una organización.

En el marco de las observaciones anteriores se plantea que la gestión se vale de varios instrumentos para poder funcionar, primeramente se hacen referencia al control y mejoramiento de los procesos, en segundo lugar, se encuentran los archivos, estos se encargarán de conservar datos y por último los instrumentos para afianzar datos y poder tomar decisiones acertadas. Es importante destacar que estas herramientas varían a lo largo de los años, por lo que no son 
estáticas, sobre todo aquellas que refieren al mundo de la informática. Es por ello que los gestores deben cambiar los instrumentos que utilizan a menudo.

Dentro del mundo empresarial la gestión presume una herramienta esencial para el desarrollo económico, ya no sólo de las propias empresas que implementan sus sistemas de gestión, igualmente para su sector de actividad o el país. (Mora, Duran, \& Zambrano, 2016)

El origen de la gestión empresarial no se puede asegurar con exactitud, pero muchos estudiosos de la historia aseveran que la gestión y administración de los recursos en una organización como una disciplina moderna, tiene sus comienzos a finales del siglo XIX e inicios del XX. (Hernández, 2011)

Para el conocimiento de la historia del umbral de la de la administración, coexisten dificultades; ciertos escritores, remontan el desarrollo de la administración a los comerciantes sumerios y a los egipcios antiguos constructores de las pirámides, o a los métodos organizativos de la Iglesia y las milicias antiguas. No obstante, muchas empresas pre-industriales, dada su escala pequeña, no se sentían obligadas a hacer frente sistemáticamente a las aplicaciones de la administración. (Hernández J. , 2013)

En este orden de idea se puede citar que las innovaciones tales como la extensión de los números árabes (entre los siglos V y XV) y la aparición de la contabilidad de partida doble en 1494 proporcionaron las herramientas para el planeamiento y el control de la organización, y de esta forma el nacimiento formal de la administración. Sin embargo es en el Siglo XIX cuando aparecen las primeras publicaciones donde se hablaba de la administración de manera científica y el primer acercamiento de un método que reclamaba urgencia dada la aparición de la Revolución Industrial.

Hace referencia la gestión empresarial a las medidas y estrategias llevadas a cabo con la finalidad de que la empresa sea viable económicamente. La misma tiene en cuenta sinfín de factores, desde lo financiero, pasando por lo productivo hasta lo logístico. Una de las principales virtudes de un hombre de negocios es la aplicación de la gestión empresarial ya que abarca a las distintas 
competencias que se deben tener para cubrir distintos flancos de una determinada actividad comercial en el contexto de una economía de mercado. (Hernández J. , 2013)

Consiguientemente, la gestión empresarial ya no es un argumento de carácter local ni tampoco nacional se debe considerar como un asunto de índole global, para el desarrollo de nuevas estrategias y la permanencia en el mercado logrando las satisfacciones de los cliente en cualquier parte del mundo. (Hernández H. , 2011)

Se precisa que el diseño de una empresa debe referir con una serie de conocimientos en numerosas áreas que puedan avalar que las condiciones de existencia de la misma se podrán desarrollar en el tiempo. Lo esencial es decretar si la actividad llevada a cabo podrá ser rentable en distintos contextos propios de la economía. Significa entonces que la gestión empresarial se enfocará en una estrategia de desarrollo que viabilice un crecimiento en función de los resultados alcanzados.

\section{Desarrollo}

Según Ucha F. (2011), refiere que la gestión empresarial es aquella actividad empresarial que, a través de diferentes individuos especializados, como pueden ser: directores institucionales, consultores, productores, gerentes, entre otros, y de acciones, buscará mejorar la productividad y la competitividad de una empresa o de un negocio.

Es importante expresar que el punto fundamental de toda la empresa es la gestión empresarial, y si se realiza adecuadamente la organización progresa, si es lo contrario la empresa declinará. (Ucha, 2011)

Hecha la observación anterior para que una gestión determinada sea recomendable y por ende brinde buenos resultados no solamente deberá hacer mejor las cosas, sino que deberá mejorar aquellas cuestiones que influyen directamente en el éxito y eso será asequible mediante la reunión de expertos que ayuden a identificar problemas, arrojen soluciones y propongan nuevas estrategias, entre otras cuestiones. En esta vertiente se deberá considerar una serie de factores, entre ellos financieros, productivos y logísticos, por citar los más importantes. Los profesionales, individuos que se dedican a la gestión empresarial, deben sí o sí dominar todos estos 
condicionantes para poder triunfar en el tema y que la empresa que dirigen sea triunfante. (López, A, \& Rubio, 2019) (Ucha, 2011)

\section{Habilidades y características gerenciales asociadas a la perdurabilidad de las empresas y a} la gestión empresarial (López, A, \& Rubio, 2019)

La perdurabilidad empresarial se logra cuando las empresas logran resultados financieros superiores y los mantienen a través del tiempo, adecuando su gestión a la intensidad de las condiciones del entorno sectorial y las fuerzas del mercado. Se refiere a desempeños eficientes en la gestión de las empresas por la coherencia en las acciones estratégicas, la identificación de necesidades del entorno y sus políticas de gobierno.

La perdurabilidad puede ser explicada por las dimensiones de dirección y gerencia, donde en la primera, se incluyen estrategia y gestión de la empresa; y en la segunda, cultura organizacional y gestión del conocimiento.

En las dimensiones de dirección y gerencia, es clave que aparezcan las habilidades y características asociadas a la perdurabilidad, algunas asociadas con rasgos demográficos como edad, experiencia laboral, nivel de educativo, entre otras; con rasgos de personalidad, con valores, y con rasgos gerenciales diferenciadores y propios del quehacer emprendedor

Según Escandón y Hurtado el número de años de experiencia laboral de los empresarios es una variable fundamental en la orientación competitiva, en la perdurabilidad de las empresas y en la gestión empresarial. Este factor permite lograr un mayor nivel de madurez en los procesos estratégicos, tácticos y operativos de las empresas según Nassif, Hashimoto y Amaral.

Rondan, Navarro y Arenas encontraron que los empresarios que logran crear empresas perdurables, son aquellos que poseen características personales distintivas, entre las cuales sobresalen la importancia que dan al orden social y a la estabilidad de la sociedad; prefieren una vida estimulante y excitante, confían más en su autoridad y en dirigir equipos de personas fundamentada en valores personales que imprimen a su gestión. 
Otros autores como Santillán, Gaona y Hernández mencionan los valores de lealtad, honestidad y sinceridad como influyentes en los ejercicios de liderazgo y gestión empresarial.

\section{Técnicas de gestión empresarial: (Figueroa)}

Las técnicas de gestión son el resultado de la convergencia de muchas tendencias, entre las que se encuentran el modelado de procesos comerciales, gestión de calidad, gestión de cambios, informática distribuida, gestión del flujo de trabajo y reingeniería de negocios.

Las técnicas de gestión (TGE) constituyen una disciplina de gestión, que requiere que los departamentos comerciales participen, por ejemplo, definiendo y cambiando las reglas comerciales. La definición de TGE como disciplina administrativa está respaldada por otros autores como Chang. Es necesario alinear los procesos comerciales con el objetivo de la organización, ya que los procesos comerciales determinan el éxito de una organización. Alinearlos con los objetivos facilita la capacidad de la organización para lograr estos objetivos.

\section{Ciclo de vida de las técnicas de gestión empresarial}

- Estrategia de proceso: desarrollo de la estrategia en nuestro ciclo de vida.

- Documentación de proceso: hemos dividido la documentación del proceso en descubrimiento (descubriendo qué procesos de negocio están disponibles) y modelado (la producción de un modelo de proceso de negocio) en nuestro ciclo de vida

- Optimización de procesos: el ciclo de análisis en el modelo de proceso de negocios en nuestro ciclo de vida indica que al analizar el modelo de proceso de negocios, se puede encontrar espacio para la mejora y es la entrada para la mejora del modelo de proceso de negocio

- Implementación del proceso: esto puede no estar relacionado con la TI, en cuyo caso se modela mediante la flecha del modelo de proceso empresarial de nuevo al proceso comercial, o relacionado con TI, en cuyo caso se sigue la flecha del diseño 
- Ejecución del proceso: hemos dividido la ejecución de sus procesos en la ejecución por el motor de ejecución del proceso de negocio (ejecución) y la interacción entre los usuarios y el motor de ejecución (la flecha de interacción)

- Control de procesos: hemos agregado monitoreo a la fase de control6.

- Herramientas de gestión empresarial

- En el sentido más amplio una herramienta de gestión es esencialmente una aplicación, solución, metodología, paradigma, método, modelo, algoritmo, procedimiento, protocolo, sistema, indicador o instrumento específico que permite y facilita la administración del negocio y la organización de manera profesional. (Garcia)

- Las empresas evolucionan de manera constante y paulatina, tanto internamente como en las relaciones establecidas con los clientes y proveedores, de forma que ha sido imprescindible la incorporación de herramientas informáticas. Es de vital importancia que las empresas se adapten e introduzcan las herramientas que se van a explicar y desarrollar a lo largo del trabajo. Cada día los mercados y negocios son más complejos haciendo necesario diseñar e implementar herramientas informáticas para que la producción de su trabajo sea más eficiente. (Garcia)

- Las herramientas informáticas en la gestión empresarial son todas aquellas que ayudan a mejorar la planificación, coordinación y gestión de la empresa mediante el uso de software o aplicaciones informáticas. Pueden ser utilizadas en cualquier departamento de la organización, controlan y mejoran las actividades de las empresas e integran todos los datos provocando que sea mejor la toma de decisiones. Es decir, mediante el uso de la informática se busca mejorar la gestión de las empresas y tener controladas todas las actividades que se llevan a cabo. Estos sistemas aceleran la comunicación tanto dentro como fuera de la empresa, generando una mejor comunicación entre la alta dirección y empleados que se encuentren en puestos de menor nivel jerárquico 
- La principal ventaja de estos sistemas es la gran ayuda aportada a los directivos para tomar decisiones ya que recoge toda la información relevante. Al recogerse en plataformas informáticas se consigue conservar la información a pesar de que se produzca rotación del personal. Por ejemplo, en una empresa hay un solo trabajador que se dedica a la gestión de un tipo de producto y sus compañeros no saben nada acerca de este tema. Si algún día dicho trabajador abandona la empresa, será más sencillo que otro empleado cubra su puesto, ya que toda la información queda recogida y podrá saber todos los temas que este ha tratado durante su experiencia laboral. (Garcia)

\section{Funciones principales de la gestión empresarial (Ucha, 2011)}

Planeación: Es una función cardinal la cual vislumbra definir las metas de la organización, establecer una estrategia global para el logro de estas metas y desarrollar una jerarquía detallada de planes para integrar y coordinar actividades, contempla las siguientes actividades

- Asignación de recursos

- Programación

Organización: Es la forma de diseñar la estructura de un negocio o empresa. Incluye la determinación de las tareas a realizar, quien las debe realizar, como se agrupan las tareas, quien reporta a quien y donde se toman las decisiones.

La estructura organizacional debe diseñarse de tal manera que quede claramente definido quien tiene que hacer determinadas tareas y quien es responsable de los resultados

Dirección conducción y liderazgo: Toda empresa, negocio, organización o institución está formada por personas, es responsabilidad de los administradores dirigir y coordinar las actividades de estas personas. La dirección consiste en motivar a los subordinados, dirigir actividades de otros (López, A, \& Rubio, 2019)

Control: por ser la última no deja de ser importante, una vez fijadas las metas, formulado los planes, delineados los arreglos estructurales, entrenado y motivado el personal, existe la posibilidad de que algo salga mal. Para asegurar que las cosas vayan como deben, se debe 
monitorear el desempeño del negocio u organización para comparar los resultados con las metas fijadas y presupuestos (Ucha, 2011).

\section{Herramientas actuales para la gestión empresarial}

De acuerdo a los razonamientos que se han venido realizando, se hace obligatorio la consideración de otro tipo de elementos de competitividad más allá de los tradicionalmente analizados. Esto determina la necesidad de contemplar nuevas técnicas, prácticas o herramientas. Entre ellas podemos ver, la cadena de valor, la reingeniería de procesos y la contabilidad estratégica (Muñoz, MR, \& Ribeiro, 2015)

\section{Contabilidad estratégica}

La contabilidad estratégica introduce una nueva visión de la contabilidad al centrar su atención en la estrategia y en la eficacia. La primera es considerada como un medio para alcanzar el liderazgo competitivo de la organización. La segunda permite maximizar los resultados de la actividad total de la organización. La contabilidad estratégica es la "única capacitada para diseñar de una manera rigurosa las estrategias más rentables y adoptar las decisiones estratégicas con el mínimo riesgo". Refieren importante investigadores que, la contabilidad directiva o estratégica presume el análisis, la ejecución y la valoración de una serie de tareas con las cuales la organización procura lograr ventajas competitivas y el éxito de la estrategia definida. Norton en su estudio define que las empresas exitosas son aquellas que proyectan, elaboran y, sobre todo, ejecutan sus estrategias con éxito, una vez establecida ésta, se requiere el seguimiento de la misma, lo que implica conocer en qué medida se han logrado los objetivos establecidos y las causas que han provocado los resultados obtenidos. Esencialmente los procesos de feedback son para detectar posibles desviaciones, dándose así la posibilidad de aplicar los mecanismos para su corrección, ya sea con el establecimiento de procesos de reingeniería, ya sea analizando cada una de las actividades de la cadena de valor. 


\section{La cadena de valor}

Muñoz MP ( ) define la cadena de valor Cito (...) como "un conjunto de actividades que se desempeñan para diseñar, producir, llevar al mercado, entregar y apoyar a sus productos" instituye una herramienta básica para analizar, identificar y potenciar las actividades de la empresa estratégicamente relevantes. Ahora bien, la cadena de valor de cada empresa constituye sólo una parte del total de la cadena de actividades creadoras de valor para el consumidor. Esto es, la cadena de valor de la empresa es sólo un eslabón dentro del conjunto de cadenas de valor que, secuencialmente, existen en un sector industrial. Por lo tanto, las actividades de la organización están enmarcadas en un conjunto más amplio de actividades denominado sistema de valor. Estas incluyen, desde el proceso de suministro de materiales utilizados por los proveedores de la organización hasta la entrega de los bienes al consumidor final (Muñoz, MR, \& Ribeiro, 2015); en esta secuencia de cadenas, el último eslabón lo conforma la cadena de valor de los clientes, y considerando que en cada una de éstas se va incorporando un valor que es, posteriormente, transmitido a la siguiente, si la empresa desea obtener y mantener una ventaja competitiva, deberá ubicar correctamente la cadena de valor de la organización en el sistema de valor. De ahí la importancia de explotar los vínculos internos y externos de la mencionada cadena. La perspectiva interna de la cadena de valor permite segmentar la empresa en sus actividades relevantes, ofreciendo la oportunidad de comprender mejor los costes y las áreas potenciales de diferenciación.

El reconocimiento de estos vínculos implica conceder la posibilidad de poder obtener una reducción en el coste total con el aumento deliberado del coste de alguna de las actividades de valor, ya sea dentro de la unidad de negocio como a través de ellas.

\section{Reingeniería de procesos}

La reingeniería de procesos, facilitada por la tecnología, surge como alternativa las estrategias de liderazgo en costes o diferenciación implantados a nivel corporativo y con las cuales las organizaciones intentan hacer frente a los cambios de su entorno. ${ }^{9}$; presume la renovación de los procesos de fabricación, haciéndolos flexibles e innovadores, al admitir el cambio oportuno de 
los métodos de producción y la reducción de tiempos y costes a lo largo de cada uno de los eslabones de la cadena de valor de la empresa. (González, Jacobo, Ochoa, \& Chairez, 2012)

La conceptualización de la reingeniería es determinada como "la revisión fundamental y el completo (radical) rediseño de los procesos para lograr mejoras sustanciales en medidas críticas del rendimiento, tales como el coste, la calidad, el servicio y la rapidez". (González, Jacobo, Ochoa, \& Chairez, 2012) ${ }^{9}$ focaliza su atención en el proceso fundamental de la organización, no en las tareas, y enlaza aquellas que sean paralelas en vez de integrar sus resultados. A través del rediseño de los procesos se espera lograr los resultados deseados, minimizar los recursos utilizados y dotar a aquellos de flexibilidad para que puedan ajustarse a las necesidades del cliente. (González, Jacobo, Ochoa, \& Chairez, 2012), (Muñoz, MR, \& Ribeiro, 2015)

\section{Otras herramientas de gestión empresarial}

Uno de los grandes impulsores de la creciente diversidad de herramientas para la gestión de la empresa, corresponde a las formas de interaccionar entre diversas plataformas informáticas heterogéneas, lo que se denomina como arquitectura orientada a servicios (SOA). Estas posibilitan que los sistemas de gestión empresariales puedan estar segmentados por módulos, cada uno de ellos con unas funciones específicas y programados con los lenguajes más adecuados para su trabajo (Baque, Chuiza, \& Pluas)

Cuando alguno de estos módulos necesita algún dato de otro, le solicita un servicio que el solicitado provee. Los siguientes enlaces muestras diferentes formas y ejemplos para la solicitud de servicios y respuestas: (SOA, SOAP, Servicio Web, XML, Middleware). (Baque, Chuiza, \& Pluas)

\section{Indicadores de Gestión}

El dato que refleja cuáles fueron las consecuencias de acciones tomadas en el pasado en el marco de una organización se conoce como indicador de gestión. La idea es que estos indicadores sienten las bases para acciones a tomar en el presente y en el futuro. Es importante que las gestiones reflejen datos veraces y fiables, ya que el análisis de la situación, de otra manera, no 
será correcto. Por otra parte, si los indicadores son ambiguos, la interpretación será complicada (Tejerina, Tilián, Jaremko, \& Party)

Lo que consiente un indicador de gestión es determinar si un proyecto o una organización están siendo exitosos o si están cumpliendo con los objetivos. El líder de la organización es quien suele establecer los indicadores de gestión, que son utilizados de manera frecuente para evaluar desempeño y resultados.

Objetivos de los indicadores (Tejerina, Tilián, Jaremko, \& Party)

Cada medidor o indicador debe satisfacer los siguientes objetivos:

- Medible: El indicador debe ser medible. Esto significa que la característica descrita debe ser cuantificable en términos ya sea del grado o frecuencia de la cantidad.

- Entendible: El indicador debe ser reconocido fácilmente por todos aquellos que lo usan.

- Controlable: El indicador debe ser controlable dentro de la estructura de la organización.

\section{Tipos de indicadores}

En el tejido de orientación hacia los procesos, un indicador puede ser de proceso o de resultados. En el primer caso, se pretende medir que está sucediendo con las actividades, y en segundo se quiere medir las salidas del proceso.

Se pueden clasificar también los indicadores en indicadores de eficacia o de eficiencia. El indicador de eficacia mide el logro de los resultados propuestos. Indica si se hicieron las cosas que se debían hacer, los aspectos correctos del proceso. Los indicadores de eficacia se enfocan en el qué se debe hacer, por tal motivo, en el establecimiento de un indicador de eficacia es fundamental conocer y definir operacionalmente los requerimientos del cliente del proceso para comparar lo que entrega el proceso contra lo que él espera.

De lo contrario, se puede estar logrando una gran eficiencia en aspectos no relevantes para el cliente. Los indicadores de eficiencia miden el nivel de ejecución del proceso, se concentran en el Cómo se hicieron las cosas y miden el rendimiento de los recursos utilizados por un proceso. Tienen que ver con la productividad. 
Otra característica deseable es la objetividad. Los indicadores deben evitar estar condicionados por factores externos, tales como la situación del país o accionar a terceros, ya sean del ámbito público o privado.

\section{Importancia de los indicadores de gestión}

- Medios, instrumentos o mecanismos para evaluar hasta qué punto o en qué medida se están logrando los objetivos.

- Permiten evaluar el desempeño de una organización frente a sus metas, objetivos y responsabilidades.

- Detectan y prevén desviaciones en el logro de los objetivos.

\section{Condiciones básicas que deben reunir los indicadores}

En primer lugar, el indicador debe ser relevante para la gestión, es decir, que aporte información imprescindible para informar, controlar, evaluar y tomar decisiones. A su vez, el cálculo que se realice a partir de las magnitudes observadas no puede dar lugar a ambigüedades. Esta condición ha de permitir que los indicadores puedan ser auditables y que se evalúe de forma externa su fiabilidad siempre que sea preciso. A esta cualidad debe añadírsele que un indicador debe ser inequívoco, es decir, que no permita interpretaciones contrapuestas. El concepto que expresa el indicador es claro y se mantiene en el tiempo. El indicador es adecuado a lo que se pretende medir (pertinencia). La información debe estar disponible en el momento en que se deben tomar las decisiones. (Tejerina, Tilián, Jaremko, \& Party)

\section{Importancia de la Gestión empresarial}

Es fundamental la gestión empresarial en lo que se refiere a la dinámica de una economía de mercado; dicha gestión se basa en la capacidad de un individuo, el empresario, de observar escenarios entro de la economía que garantizan una rentabilidad razonable como para generar en los mismos bienes y servicios. Significando que recae en el empresario el rol de dar cuenta de oportunidades económicas y a partir de esta visión movilizar al capital hacia las mismas. Este fenómeno es clave en el capitalismo y ha sido puesto en relieve en distintos textos teóricos de 
diversos economistas, destacándose entre todos ellos los de Joseph. (Importancia de la gestión empresarial. )

Por lo tanto la gestión empresarial es la que atestigua que la oferta cubra a la demanda en distintas actividades productivas, generando además procedimientos que hacen que los costos bajen continuamente y beneficien al consumidor. Gracias a esta actividad, el capital se orienta siempre a producir aquello que la sociedad demanda para la satisfacción de determinadas necesidades, circunstancia que da cuenta de la eficiencia del mercado en lo que respecta a asignación de recursos.

\section{La gestión empresarial es importante porque:}

Ayuda a alcanzar objetivos comunes: logra al organizar los factores de producción e integrar los recursos de manera efectiva para alcanzar objetivos. Dirige los esfuerzos del equipo hacia el cumplimiento de estos objetivos predeterminados. Una definición clara de los objetivos evita desperdiciar tiempo, dinero y esfuerzo (Caraballo).

\section{Contribuye a una utilización óptima de los recursos}

La bienhechora gestión empresarial manipula los recursos humanos y físicos de manera productiva. Se debe optimizar el uso de recursos escasos y seleccionar su mejor uso, y para ello, valerse de expertos, profesionales que aporten sus habilidades y conocimiento en una utilización correcta y libre de desperdicios de estos recursos. Si los empleados y las máquinas trabajan al máximo de su capacidad no nos encontraremos con situaciones de subempleo. (Caraballo),

\section{Reduce costes}

Adquiere máximos efectos con una mínima inversión, debido a una planificación correcta que permita invertir poco y conseguir mucho. Como resultado una buena gestión empresarial debe orientarse a utilizar los recursos físicos, humanos y financieros de tal manera que se encuentre la mejor combinación, lo cual debería ayudar a recortar tus costes. 


\section{Referencias}

1. Mora LL, Duran ME, Zambrano JG. Consideraciones actuales sobre gestión empresarial. Rev Dom. Cien.2016. [Citado 2 Oct 2019]; 2(4); Disponible en: https://www.google.com/url?q=https://dialnet.unirioja.es/descarga/articulo/5802891.pdf\& sa=U\&ved=2ahUKEwjv2KL876HlAhUkqlkKHXb6BJIQFjAFegQICBAB\&usg=AOvVa w0GZu8izW8TUdT4U0yvNayq

2. Hernández HG. La gestión empresarial, un enfoque del siglo $\mathrm{XX}$, desde las teorías administrativas científica, funcional, burocrática y de relaciónes humanas. Rev Escenarios. 2011. [Citado 2 Oct 2019]; 9(1); Disponible en: https://www.google.com/url?q=https://dialnet.unirioja.es/descarga/articulo/3875234.pdf\& sa=U\&ved=2ahUKEwjv2KL876HlAhUkqlkKHXb6BJIQFjAEegQICRAB\&usg=AOvVa w2IysS5Pd2W1lte1K14pTSf

3. Hernández J. Modelo de gestión empresarial según nuevas tendencias: intangibles y calidad total. Aplicación al sector camaronero de Venezuela. [Citado 2 Oct 2019]. Disponible

en: https://www.google.com/url?q=https://helvia.uco.es/bitstream/handle/10396/10762/20130 00000806.pdf\%3Fsequence\%3D1\%26isAllowed\%3Dy\&sa=U\&ved=2ahUKEwi6wa2_8a HlAhXP1lkKHZxwChkQFjAHegQIARAB\&usg=AOvVaw0K2QzNA9u531BBvkh87HK

4. Fidalgo A. Definición de Gestión Empresarial. [Citado 2 Oct 2019]. Disponible en: https://www.definicionabc.com/economia/gestion-empresarial.php

5. López L, Parra A, Rubio G. Habilidades gerenciales y su relación con la perdurabilidad de las empresas: estudios de caso empíricos en Florencia e Ibagué (Colombia). Rev Espacios. 2019. [Citado 2 Oct 2019]; 40(11); Disponible en: https://www.google.com/url?sa=t\&source=web\&cd=9\&ved=2ahUKEwiY8bjsyKblAhVn x1kKHRsJAa8QFjAIegQICBAB\&url=http\%3A\%2F\%2Fwww.revistaespacios.com\%2Fa 19v40n11\%2Fa19v40n11p05.pdf\&usg=AOvVaw397yW2cMVzwUhAoy1_Y0FO 
6. Figueroa D. Técnicas de gestión empresarial. [Citado 2 Oct 2019]. Disponible en: https://www.google.com/url?sa=t\&source=web\&cd=9\&ved=2ahUKEwjPl4P1yablAhUQ 01kKHZIRAFIQFjAIegQIAhAB\&url=https\%3A\%2F\%2Frepository.unimilitar.edu.co\%2 Fbitstream\%2F10654\%2F18027\%2F1\%2FFigueroaPenillaDavidSteven2018.pdf.pdf\&usg =AOvVaw0AzwSA_XHn70XlCvadiiLA

7. García S. Herramientas informáticas en la gestión empresarial. [Citado 2 Oct 2019]. Disponible en: https://www.google.com/url?sa=t\&source=web\&cd=8\&ved=2ahUKEwi415_yablAhXQxlkKHS2DDy0QFjAHegQICBAB\&url=https\%3A\%2F\%2Fbiblioteca.unirioj a.es\%2Ftfe_e\%2FTFE002513.pdf\&usg=AOvVaw2LJfibSDkSNDyhFAwcAU7a

8. Muñoz MP, Cabrita MR, Ribeiro da Silva ML, Diéguez G. Técnicas de gestión empresarial en la globalización. Revista de Ciencias Sociales. 2015. [Citado 2 Oct 2019]; XXI (3); Disponible en: https://www.google.com/url?q=http://www.redalyc.org/pdf/280/28042299006.pdf\&sa=U \&ved=2ahUKEwjT76nU9aHlAhXkqFkKHeuICx4QFjAEegQICBAB\&usg=AOvVaw1q3 2JUDIP9-ok2FArfO_aG

9. González NE, Jacobo CA, Ochoa B, Chairez EK. Gestión empresarial, oportunidad de mejoras en los negocios. [Citado 9 Oct 2019]. Disponible en: https://www.google.com/url?q=https://www.mincetur.gob.pe/wpcontent/uploads/documentos/comercio_exterior/Sites/ueperu/consultora/docs_taller/Prese ntaciones_Tumbes_y_Piura/1.2.1.2.F1\%2520Gestion_Empresarial\%252020080912.pdf\& sa=U\&ved=2ahUKEwjv2KL876HlAhUkqlkKHXb6BJIQFjAHegQIChAB\&usg=AOvVa w1I6Xrdt-JTbsBZSaM70KAL

10. Baque E, Chuiza A, Pluas G. Análisis de los cierres de cooperativas de ahorro y crédito en el año 2012. Diseño de estrategias de coaching ejecutivo para directivos. [Citado 10 Oct 2019]. Disponible en: https://www.google.com/url?q=http://repositorio.ug.edu.ec/bitstream/redug/13669/1/TESI S\%2520- 


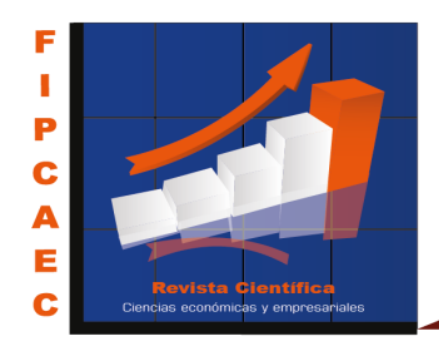

\%2520COACHING\%2520EJECUTIVO.pdf\&sa=U\&ved=2ahUKEwi6wa2_8aHlAhXP11 kKHZxwChkQFjACegQIChAB\&usg=AOvVaw3rZNP_2Kysc0KRkPRoS-Ac

11. Tejerina AE, Tilián CE, Jaremko OA, Party MG. Recopilación de bibliografía y material de estudios realizada por integrantes de la cátedra Gestión de Empresas. [Citado 10 Oct 2019].

Disponible

en: https://www.google.com/url?q=http://www.economicas.unsa.edu.ar/adminperso/guias.pdf \&sa=U\&ved=2ahUKEwjv2KL876HlAhUkqlkKHXb6BJIQFjAJegQIBxAB\&usg=AOvV aw0NYVmFsGeIbXTSjILqsb1h

12. Ruiz D. Técnicas de gestión empresarial aplicadas a organizaciones sociales. [Citado 11 Oct 2019]. Disponible en: https://www.google.com/url?q=http://www.eumed.net/ce/2004/drmgu.pdf\&sa=U\&ved=2ahUKEwjT76nU9aHlAhXkqFkKHeuICx4QFjABegQIChAB\&usg= AOvVaw0iuoAdjU43iLhzbrkJo66g

13. Millien O. Importancia de la gestión empresarial. [Citado 11 Oct 2019]. Disponible en: https://www.importancia.org/gestion-empresarial.php

14. Caraballo L. La Importancia de la gestión empresarial. [Citado 12 Oct 2019]. Disponible en: https://www.sage.com/es-es/blog/la-importancia-de-la-gestion-empresarial/

15. Castillo M. ¿Será importante la gestión empresarial? [Citado 12 Oct 2019]. Disponible en: https://aplimedia.com/la-importancia-de-la-gestion-empresarial/

\section{References}

1. Mora LL, Duran ME, Zambrano JG. Current considerations on business management. Rev Dom. One Hundred. 2016. [Quoted 2 Oct 2019]; 2 (4); Available at: https://www.google.com/url?q=https://dialnet.unirioja.es/descarga/articulo/5802891.pdf\& sa=U\&ved=2ahUKEwjv2KL876HlAhUkqlkKHXb6BJIQFjAFegQICBAB\&usg0AOvdU 8QU0dU8dU0db04dz03 
2. Hernández HG. Business management, an approach of the twentieth century, from the administrative theories scientific, functional, bureaucratic and human relations. Rev Scenarios. 2011. [Quoted 2 Oct 2019]; 9 (1); Available at: https://www.google.com/url?q=https://dialnet.unirioja.es/descarga/articulo/3875234.pdf\& sa=U\&ved=2ahUKEwjv2KL876HlAhUkqlkKHXb6BJIQFjAEegQICRAB\&usg2AOvVd dd1d1d1d1d1d1d1

3. Hernández J. Business management model according to new trends: intangibles and total quality. Application to the shrimp sector of Venezuela. [Quoted 2 Oct 2019]. Available at: https://www.google.com/url?q=https://helvia.uco.es/bitstream/handle/10396/10762/20130 00000806.pdf\%3Fsequence\%3D1\%26isAllowed\%3Dy\&sa=U\&ved=2ahUKEwi6wa2_8a HlAhXP1lkKgQjABAHKgQxABAH = AOvVaw0K2QzNA9u531BBvkh87HK

4. Fidalgo A. Definition of Business Management. [Quoted 2 Oct 2019]. Available at: https://www.definicionabc.com/economia/gestion-empresarial.php

5. López L, Parra A, Rubio G. Management skills and their relationship with the endurance of companies: empirical case studies in Florencia and Ibagué (Colombia). Rev Spaces. 2019. [Quoted 2 Oct 2019]; 40 (11); Available at: https://www.google.com/url?sa=t\&source=web\&cd=9\&ved=2ahUKEwiY8bjsyKblAhVn x1kKHRsJAa8QFjAIegQICBAB\&url=http\%3A\%2F\%2Fwww.revistaespacios.com\%2Fa 19v40n11\%2Fa19v40n11p05.pdf\&usg=AOvVaw397yW2cMVzwUhAoy1_Y0FO

6. Figueroa D. Business management techniques. [Quoted 2 Oct 2019]. Available at: https://www.google.com/url?sa=t\&source=web\&cd=9\&ved=2ahUKEwjPl4P1yablAhUQ 01kKHZIRAFIQFjAIegQIAhAB\&url=https\%3A\%2F\%2Frepository.unimilitar.edu.co\%2 Fbitstream\%2F10654\%2F18027\%2F1\%2FFigueroaPenillaDavidSteven2018.pdf .pdf \& usg = AOvVaw0AzwSA_XHn70XICvadiiLA

7. García S. Computer tools in business management. [Quoted 2 Oct 2019]. Available at: https://www.google.com/url?sa=t\&source=web\&cd=8\&ved=2ahUKEwi415_yablAhXQxlkKHS2DDy0QFjAHegQICBAB\&url=https\%3A\%2F\%2Fbiblioteca.unirioj a.es\%2Ftfe_e\%dFawdw2dw2dw2dw2dw2dw2dw2dw2dw2dw3d2db3db3db3a03db3aw0 


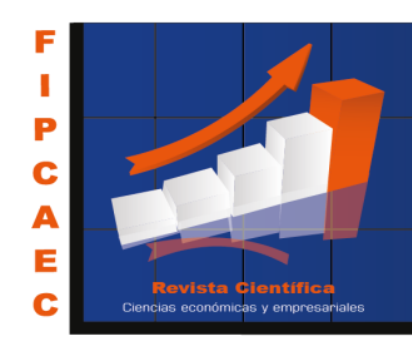

4aw02aw02aw02aw02aw02aw03aw02aw02aw02aw02aw02aw02aw02aw02aw02aw02a w02aw02aw02aw02aw02aw02aw02aw02aw04aw04aw04aw04aw04aw04aw04a4

8. Muñoz MP, Cabrita MR, Ribeiro da Silva ML, Diéguez G. Techniques of business management in globalization. Social Sciences Magazine. 2015. [Quoted 2 Oct 2019]; XXI (3); Available

at: https://www.google.com/url?q=http://www.redalyc.org/pdf/280/28042299006.pdf\&sa=U \&ved=2ahUKEwjT76nU9aHIAhXkqFkKHeuICx4QFjAEegQICBAB\&usg=AOvVaw1OvVaw1-OvVaw1-OvVaw1-OvVaw1-OvVaw1-O2J

9. González NE, Jacobo CA, Ochoa B, Chairez EK. Business management, opportunity for business improvements. [Quoted 9 Oct 2019]. Available at: https://www.google.com/url?q=https://www.mincetur.gob.pe/wpcontent/uploads/documentos/comercio_exterior/Sites/ueperu/consultora/docs_taller/Prese ntaciones_Tumbes_y_Piura/1.2 .1.2.F1\% 2520 Management_Empresarial\% 252020080912.pdf $\quad \& \quad$ sa $=\quad \mathrm{U} \quad \& \quad$ ved $=$ 2ahUKEwjv2KL876HlAhUkqlkKHXb6BJIQFjAHegQIChAB \& usg = AOvVaw1I6Xrdt-JTbsBZSaM

10. Baque E, Chuiza A, Pluas G. Analysis of savings and credit cooperative closures in 2012. Design of executive coaching strategies for managers. [Quoted 10 Oct 2019]. Available at:

https://www.google.com/url?q=http://repositorio.ug.edu.ec/bitstream/redug/13669/1/TESI S\%2520-\%2520COACHING\%2520EJECUTIVO.pdf\&sa=U\&ved= 2ahUKEwi6wa2_8aHlAhXP1lkKHZxwChkQFjACegQIChAB \& usg = AOvVaw3rZNP_2Kysc0KRkPRoS-Ac

11. Tejerina AE, Tilián CE, Jaremko OA, Party MG. Compilation of bibliography and study material carried out by members of the Business Management Chair. [Quoted 10 Oct 2019]. Available at: https://www.google.com/url?q=http://www.economicas.unsa.edu.ar/adminperso/guias.pdf 
\&sa=U\&ved=2ahUKEwjv2KL876HlAhUkqlkKHXb6BJIQFjAJegQIBxAB\&usg0AevVa xsjbbjsb1JebVsb1JebVsb1JeVVSG0NGVGSLVG

12. Ruiz D. Business management techniques applied to social organizations. [Quoted 11 Oct 2019]. Available at: https://www.google.com/url?q=http://www.eumed.net/ce/2004/drmgu.pdf\&sa=U\&ved=2ahUKEwjT76nU9aHlAhXkqFkKHeuICx4QFjABegQIChAB\&usg= AOvVaw0iuoLdjU43Jo66

13. Millien O. Importance of business management. [Quoted 11 Oct 2019]. Available at: https://www.importancia.org/gestion-empresarial.php

14. Caraballo L. The Importance of business management. [Cited 12 Oct 2019]. Available at: https://www.sage.com/es-es/blog/la-importancia-de-la-gestion-empresarial/

15. Castillo M. Will business management be important? [Cited 12 Oct 2019]. Available at: https://aplimedia.com/la-importancia-de-la-gestion-empresarial/

(C2018 por el autor. Este artículo es de acceso abierto y distribuido según los términos y condiciones de la licencia Creative Commons Atribución-NoComercial-Compartirlgual 4.0 Internacional (CC BY-NC-SA 4.0) (https://creativecommons.org/licenses/by-nc-sa/4.0/). 